\title{
Article \\ Effects of Metal Boundary Stretching and Sample Translational Motion on Microwave Heating
}

\author{
Hao Gu ${ }^{1}$, Jinghua Ye ${ }^{2}$, Ge Wang ${ }^{1}$, Xueting Yan ${ }^{1}$, Huacheng Zhu ${ }^{1}\left(\mathbb{D}\right.$ and Yang Yang ${ }^{1, *(\mathbb{D}}$ \\ 1 College of Electronic and Information Engineering, Sichuan University, Chengdu 610065, China; \\ guhao@stu.scu.edu.cn (H.G.); wangge@stu.scu.edu.cn (G.W.); xuetingY1222@163.com (X.Y.); \\ hczhu@scu.edu.cn (H.Z.) \\ 2 School of Electronic Information and Electrical Engineering, Chengdu University, Chengdu 610100, China; \\ yejinghua@cdu.edu.cn \\ * Correspondence: yyang@scu.edu.cn; Tel.: +86-28-8547-0659
}

Citation: Gu, H.; Ye, J.; Wang, G.; Yan, X.; Zhu, H.; Yang, Y. Effects of Metal Boundary Stretching and Sample Translational Motion on Microwave Heating. Processes 2022, 10, 246 https://doi.org/10.3390/pr10020246

Academic Editor: Clayton Jeffryes

Received: 21 December 2021

Accepted: 24 January 2022

Published: 27 January 2022

Publisher's Note: MDPI stays neutral with regard to jurisdictional claims in published maps and institutional affiliations.

Copyright: (C) 2022 by the authors. Licensee MDPI, Basel, Switzerland. This article is an open access article distributed under the terms and conditions of the Creative Commons Attribution (CC BY) license (https:// creativecommons.org/licenses/by/ $4.0 /)$.

\begin{abstract}
For the purpose of improving uniformity and efficiency of microwave heating, moving components are widely used. In this paper, a kind of stretching microwave oven with a conveyor belt is designed. The conveyor belt and the stretching motion of the upper surface of the microwave oven make the electric field in the microwave cavity continuously change during heating, so that the absorption pattern of materials does not remain constant. The transformation optics method is used to simulate the stretching motion of the upper wall of the microwave oven, and the implicit function method is used to simulate the translational motion of the sample on the conveyor belt. The correctness of the simulation model is validated by experiments. The heating effects for the cases of fixed heating, stretching and translational motion are compared. Finally, the heating effects for the proposed model with different heated materials are also discussed.
\end{abstract}

Keywords: microwave heating; heating uniformity; stretching motion; conveyor belt

\section{Introduction}

Microwaves have been widely used in radar, navigation, food processing, chemical production, sewage treatment, biomedical and other fields [1] for their high efficiency and environmental friendliness. Microwaves have been used to heat food for decades, and the microwave oven is one of the most common household appliances [2]. Different from traditional heating methods, microwave heating has the advantages of selective heating, fast heating and energy conservation [3]. However, nonuniformity is one of the main disadvantages of microwave heating [4]. In order to overcome this significant defect, the most common method is adding moving elements such as turntable and mode stirrer in the microwave oven $[5,6]$.

The size of the microwave oven is usually fixed, and the electromagnetic wave in the cavity is usually in the form of a standing wave [7]. For the purpose of improving the uniformity of microwave heating, it is common to adjust the absorption mode of the microwave energy of the heated materials by changing the distribution of the electromagnetic field inside the microwave cavity and the position of the heated materials [8,9]. Changing the distribution of the electromagnetic field inside the microwave oven is usually achieved by changing the microwave frequency, phase and position of the feed port [10-13]. For example, for causing random scattering of microwaves, conductive beads are used [14,15], and a mode stirrer is introduced to stir the distribution of the electric field in the microwave oven $[5,16]$. To change the position of the heated material, a turntable and conveyor belt are often used inside the microwave oven $[17,18]$. By constantly optimizing the microwave cavity structure during heating, the electric field distribution inside the microwave cavity will be more uniform, thus the uniformity of microwave heating can be improved $[19,20]$. 
The size and form of the heated materials are also studied by some researchers with the aim of improving uniformity [21,22].

Plaza-Gonzalez et al. $[16,23]$ analyzed the impact of various mode stirrers with distinct structures and the distribution of the electric field inside the microwave oven and determined that the motion of mode stirrers can significantly improve the uniformity of the heated substances. Ye et al. found that the closer the distance between the mode stirrers and the sample, the more effect on improving the heating uniformity [5]. The effects of the size of the microwave cavity and the phase of the feeding port on heating uniformity are also studied by D Luan et al. [7], and the results show that, by these two methods, the heating uniformity is significantly improved. Ma et al. [24] put the material on a transmission device for microwave processing, in this way, the electric field pattern inside the material changes with time. Zhu et al. [8] presented a new type of microwave cavity structure with rotary radiation, and the results show that the structure with rotary radiation has obvious advantages for improving the heating uniformity. He et al. [25] proposed a two-port rotating feed structure and studied the effect of a rectangular port rotation angle on material heating uniformity. Although moving components have been widely used in microwave heating, the simulation becomes more complicated due to the complex mathematical modeling and the constant repartitioning of the mesh structure.

Changing the cavity structure continuously can improve heating uniformity, but similar to the mode stirrers, heating uniformity based only on the stretching motion of a surface in the cavity has a limited effect, and the conveyor belt can only improve the heating uniformity in one direction. Instead of just stretching the upper surface of the cavity, the heated sample with the addition of a conveyor belt may have more heating location choices, which means the combination of the two modes of motion may have better effects on the improvement of heating uniformity. Moreover, in order to solve the simulation problem of the cavity model with two motion modes proposed in this paper, the co-simulation based on implicit function method and transform optics method is first proposed, and the coupling degree of the two methods is verified by experiments. Based on this, the purposes of this paper are summarized as follows:

(1) design a microwave oven with stretchable upper surface and conveyor belt, and by stretching and translational motion, improving the heating uniformity and efficiency;

(2) use the transformation optics method to solve the calculation of stretching motion, and use the implicit function method to solve the calculation of translational motion;

(3) sensitivity analysis: analyze the influence of translational motion, stretching motion and select location heating on the uniformity and efficiency of microwave heating, as well as the heating performance of different materials in the proposed cavity.

\section{Methodology}

\subsection{Model Description}

In this paper, we build a 3-D simulation model of the proposed microwave oven by COMSOL Multiphysics software, as shown in Figure 1. The structure of proposed model is divided into two parts: the upper layer and the lower layer. The upper layer is a transformation optical layer with a height of $40 \mathrm{~mm}$ and a length and width of $240 \mathrm{~mm}$. The stretching motion of the upper wall of the microwave oven is replaced by a change of the parameters including the dielectric coefficient and permeability of the optical layer, and the stretching range is $0 \mathrm{~mm}$ to $100 \mathrm{~mm}$. The size of the lower layer cavity is $240 \mathrm{~mm} \times 240 \mathrm{~mm} \times 150 \mathrm{~mm}$, namely, the height range of the microwave oven is $150 \mathrm{~mm}$ to $250 \mathrm{~mm}$. The cavity is fed by a standard WR-340 rectangular waveguide feeding microwave in $\mathrm{TE}_{10}$ mode at a frequency of $2.45 \mathrm{GHz}$ from the port. There is a polytetrafluoroethylene conveyor belt (with a thickness of $5 \mathrm{~mm}$, a length of $240 \mathrm{~mm}$, and a width of $50 \mathrm{~mm}$ ) inside the cavity that can be shifted. On the conveyor belt, the heated material moves in translational motion. 


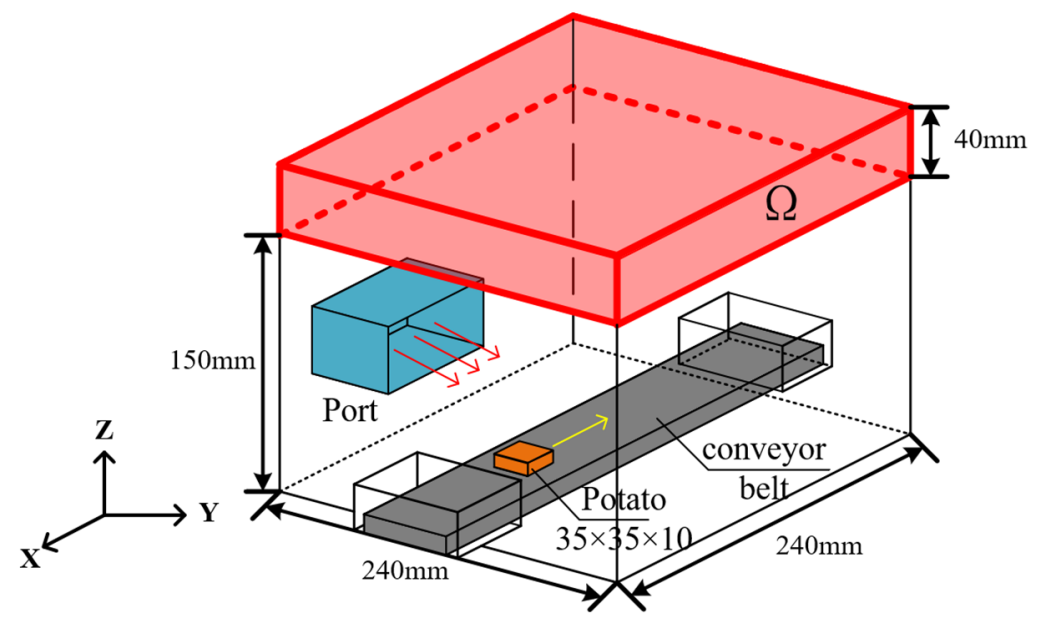

Figure 1. Structure of the simulation model. (Unit: $\mathrm{mm}$ ).

\subsection{Parameters}

Table 1 shows the input parameters of the proposed model. The ambient temperature is set at $20^{\circ} \mathrm{C}$. Due to the relative permittivity of potatoes changing with temperature, it is set as a function of temperature.

Table 1. Material properties in physical domains of the proposed model.

\begin{tabular}{|c|c|c|c|}
\hline Property & Physical Domains & Value & Source \\
\hline \multirow{5}{*}{$\begin{array}{l}\text { Dielectric constant } \\
\qquad\left(\varepsilon^{\prime}\right)\end{array}$} & Potato & $-6.4 \times 10^{-3} \mathrm{~T}^{2}+2 \times 10^{-1} \mathrm{~T}+56.8$ & [26] \\
\hline & Air & 1 & COMSOL Built-in \\
\hline & Conveyor belt & 4.2 & COMSOL Built-in \\
\hline & Beef & 40 & [27] \\
\hline & Distilled water & 78 & COMSOL Built-in \\
\hline \multirow{6}{*}{ Dielectric loss $\left(\varepsilon^{\prime \prime}\right)$} & Air & 0 & COMSOL Built-in \\
\hline & Potato & $-10^{-4} \mathrm{~T}^{2}-1.08 \times 10^{-1} \mathrm{~T}+16.1$ & [26] \\
\hline & Conveyor belt & 0 & COMSOL Built-in \\
\hline & Beef & 12 & [27] \\
\hline & Distilled water & 10 & COMSOL Built-in \\
\hline & Air & 1 & COMSOL Built-in \\
\hline \multirow{4}{*}{ Relative permeability } & Potato & 1 & [26] \\
\hline & Conveyor belt & 1 & COMSOL Built-in \\
\hline & Beef & 1 & [27] \\
\hline & Distilled water & 1 & COMSOL Built-in \\
\hline \multirow{3}{*}{$\begin{array}{l}\text { Thermal conductivity } \\
\qquad(\mathrm{W} / \mathrm{m} \cdot \mathrm{K})\end{array}$} & Potato & 0.64 & [26] \\
\hline & Beef & 0.51 & [27] \\
\hline & Distilled water & 0.59 & COMSOL Built-in \\
\hline \multirow{3}{*}{ Density $\left(\mathrm{kg} / \mathrm{m}^{3}\right)$} & Potato & 1050 & {$[26]$} \\
\hline & Beef & 1076 & [27] \\
\hline & Distilled water & 1000 & COMSOL Built-in \\
\hline \multirow{3}{*}{$\begin{array}{c}\text { Specific heat } \\
\text { capacity }(\mathrm{J} / \mathrm{kg} \cdot \mathrm{K})\end{array}$} & Potato & 3640 & [26] \\
\hline & Beef & 3430 & [27] \\
\hline & Distilled water & 4187 & COMSOL Built-in \\
\hline
\end{tabular}

\subsection{Governing Equations}

2.3.1. Governing Equations of Electromagnetic Field and Heat Transfer

Generally, by solving Maxwell's wave equations, the electric field distribution in the microwave cavity is obtained [28]:

$$
\nabla \times \mu_{r}^{-1}(\nabla \times \mathbf{E})-k_{0}^{2}\left(\varepsilon_{r}-\frac{j \sigma}{\omega \varepsilon_{0}}\right) \mathbf{E}=0
$$

where $\mathbf{E}$ is the electric field; $\varepsilon_{0}$ denotes the permittivity of vacuum; $\sigma$ is the electrical conductivity; $\mu_{r}$ denotes the relative permeability; $\varepsilon_{r}$ is the relative permittivity; $k_{0}$ represents the wave number in the vacuum. 
Based on the calculation of the electric field distribution, furthermore, the heat transfer process is computed, and during the microwave heating process, electric field and heat transfer process are coupled. In general, by solving the heat transfer equation, the distribution of heated material is obtained [6]:

$$
\rho C_{p} \frac{\partial T}{\partial t}-\nabla \cdot(k \nabla T)=Q_{e}
$$

where $\rho, C_{p}, k$ and $Q_{e}$ denote the density of heated material, the heat capacity, the thermal conductivity and the heat source respectively. During the microwave heating process, the dissipated power of the microwave in the heated material as the heat source is obtained from the electric field, and $Q_{e}$ can be expressed as [6]:

$$
Q_{e}=\frac{1}{2} \omega \varepsilon_{0} \varepsilon^{\prime \prime}|\mathbf{E}|^{2}
$$

where $\omega, \varepsilon_{0}, \varepsilon^{\prime \prime}$ are angular frequency of microwave, the relative permittivity of vacuum and the imaginary part of the dielectric relative permittivity of heated sample respectively.

\subsubsection{Boundary Conditions}

In addition to the feeding port, the whole cavity shell is considered as a perfect electric conductor as shown in Figure 1, which means the tangential component of the electric field is zero:

$$
\mathbf{n} \times \mathbf{E}=0
$$

where $\mathbf{n}$ denotes the unit normal vector of the corresponding wall.

Inside the microwave cavity, with respect to thermal conditions, by convection the surfaces of heated materials exchange heat with the surrounding air, which can be defined by [6]:

$$
-k \frac{\partial T}{\partial \mathbf{n}}=h \cdot\left(T-T_{a i r}\right)
$$

where $\mathbf{n}$ is the direction normal to the surface of heated sample, $h$ and $T_{\text {air }}$ represent the heat transfer coefficient and the temperature of air respectively, $T$ denotes the point temperature of heated sample which contacts with air. The value of $h$ is set as $10 \mathrm{~W} /\left(\mathrm{m}^{2} \cdot \mathrm{K}\right)$ in this study. The initial temperature of heated materials and air are both $293.15 \mathrm{~K}\left(20^{\circ} \mathrm{C}\right)$. The thermal boundary between heated materials and the conveyor belt is set as insulation boundary condition due to the short heating time.

\subsubsection{Transformation Optics Method for the Computation of Stretching Motion}

In the proposed model, the mesh change caused by the stretching of the microwave upper wall makes the simulation more complex. Fortunately, the transformation optics method can solve this problem effectively. Since Maxwell's equations still retain their form after coordinate transformation $[29,30]$, namely, through some coordinate transformation, the medium parameters in the transformation region can be transformed into tensor form to realize the preset electromagnetic field transformation, so as to be equivalent to the movement of the object. In this way, the mesh redivision caused by the object movement is avoided and the complexity of the calculation is reduced. Since the upper wall of the proposed model only moves in the Z-axis direction, the coordinate transformation is simple; the following describes the coordinate transformation and the medium transformation process of the transformation area.

Figure 2 is the diagram of coordinate transformation of the 2-D cross section of the proposed cavity model. Region $\Omega$ in Figure $2 \mathrm{a}$ is the transformation optical region, and the stretching motion of the upper wall of the cavity is realized by transforming its medium parameters into the tensor form according to the corresponding coordinate transformation. 
Figure $2 \mathrm{~b}$ depicts the actual stretched position of the upper wall of the microwave oven (The coordinate of the upper surface is $Z_{c}$ ). The coordinate transformation is set as [27]:

$$
\begin{gathered}
x^{\prime}=x \\
y^{\prime}=y \\
z^{\prime}=z_{a}+\frac{z_{b}-z_{a}}{z_{c}-z_{a}}\left(z-z_{a}\right)
\end{gathered}
$$

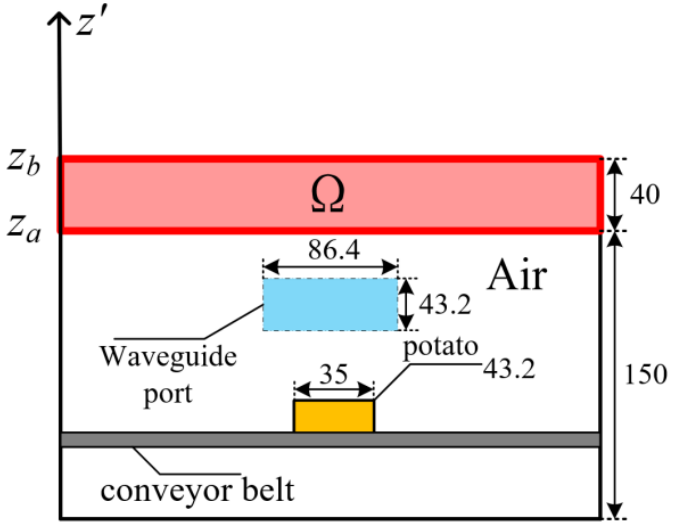

(a)

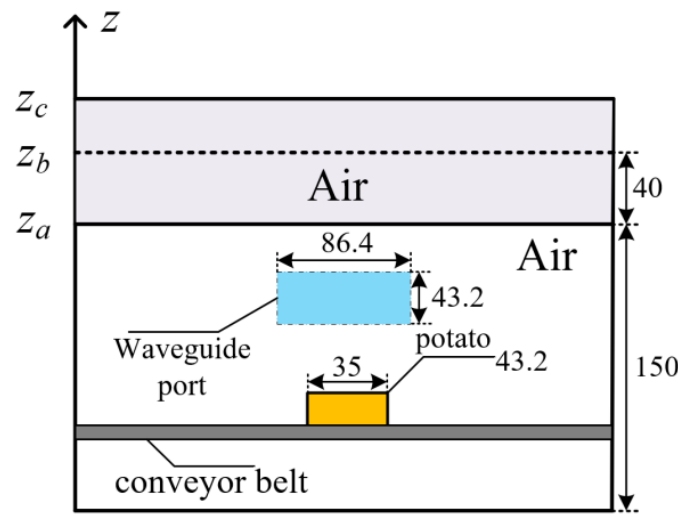

(b)

Figure 2. The diagram of coordinate transformation of the 2-D cross section of the proposed cavity model. (a) transformation optical model (b) actual stretched position with original coordinate system.

Based on the coordinate transformation equations, the tensor parameters in the optical transformation region $\Omega$ can be obtained [27]:

$$
\begin{aligned}
& \overline{\bar{\varepsilon}}=\varepsilon \overline{\bar{\eta}} \\
& \overline{\bar{\mu}}=\mu \overline{\bar{\eta}}
\end{aligned}
$$

where:

$$
\overline{\bar{\eta}}=\overline{\bar{J}}^{T} \cdot \overline{\bar{J}} / \operatorname{det} \overline{\bar{J}}
$$

$\overline{\bar{J}}$ is the Jacobian tensor:

$$
\overline{\bar{J}}=\left[\begin{array}{lll}
\partial x^{\prime} / \partial x & \partial x^{\prime} / \partial y & \partial x^{\prime} / \partial z \\
\partial y^{\prime} / \partial x & \partial y^{\prime} / \partial y & \partial y^{\prime} / \partial z \\
\partial z^{\prime} / \partial x & \partial z^{\prime} / \partial y & \partial z^{\prime} / \partial z
\end{array}\right]
$$

Combining Equations (6)-(8) with (12), $\overline{\bar{\eta}}$ can be obtained:

$$
\begin{gathered}
\overline{\bar{\eta}}=\left[\begin{array}{ccc}
1 / k & 0 & 0 \\
0 & 1 / k & 0 \\
0 & 0 & k
\end{array}\right] \\
k=\frac{z_{b}-z_{a}}{z_{c}-z_{a}}
\end{gathered}
$$

It is worth noting that coordinate transformation only occurs in the region where $z$ coordinates are greater than $Z_{a}$, that is to say, the parameters of the region where the heated material and the conveyor belt are not affected by coordinate transformation. 


\subsubsection{Implicit Method for the Computation of Translational Motion}

In addition to the stretching motion of the upper wall of the cavity, there is also a conveyor belt of translational motion in the cavity. Similarly, due to the translational movement of the heated material, the mesh will be redivided along with the movement, increasing the amount of computation and, worse, leading to significant simulation errors. Fortunately, the implicit function method can efficiently solve the translational movement calculation of conveyor belt [31]. The implicit function method sets the parameters of the moving region as functions related to spatial position and time, that is, the motion of the heated material is represented by the changes of its parameters with respect to space and time. In the proposed model, schematic diagrams of 3-D and 2-D conveyor belt areas are shown in Figure 3.

(a)

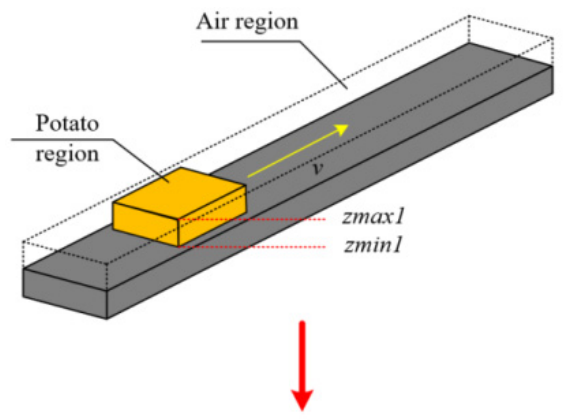

(b)

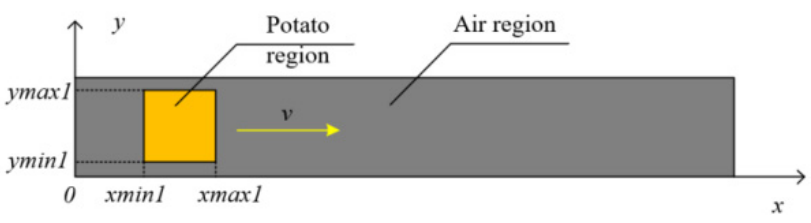

Figure 3. The schematic diagrams of conveyor belt areas: (a) 3-D diagram; (b) 2-D diagram.

First, a step equation is defined to distinguish the physical domain interior and exterior:

$$
H(D)= \begin{cases}1, & D \geq 0 \\ 0, & D<0\end{cases}
$$

where $D$ is the implicit function of a different physical domain.

Based on this, we set the dielectric constant of the moving region as follows:

$$
\varepsilon=\varepsilon_{p} \cdot H\left(D_{p}(x, y, z, t)\right)+\varepsilon_{a i r} \cdot H\left(D_{a i r}(x, y, z, t)\right)
$$

where $\varepsilon_{p}, \varepsilon_{\text {air }}$ denote the permittivity of the heated material and air respectively. $D_{p}(x, y, z, t)$, and $D_{\text {air }}(x, y, z, t)$ represent the implicit function of the potato region and air region, respectively. Next, set the implicit functions for each region:

$$
\begin{gathered}
D_{p}(x, y, z, t)=\min \left(\begin{array}{ccc}
x-x_{\min 1}(t), & y-y_{\min 1} & z-z_{\min 1} \\
x_{\max 1}(t)-x, & y_{\max 1}-y, & z_{\max 1}-z
\end{array}\right)>0 \\
x_{\min 1}(t)=x_{\min 1}+v t \\
x_{\max 1}(t)=x_{\max 1}+v t \\
D_{\text {air }}(x, y, z, t)=1-D_{p}(x, y, z, t)
\end{gathered}
$$

After setting the implicit function of each physical region, Equation (1) is solved in combination with the parameter setting of the transformation optical layer, realizing the calculation of electric field distribution at the corresponding movement position of the conveyor belt and the stretching height. 


\subsection{Mesh Setting}

Appropriate mesh type and size can improve the simulation accuracy and reduce the simulation time, so it is necessary to conduct an independent study on the mesh. The normalized power absorption (NPA) is used to select an appropriate mesh, which is defined as the power absorbed by processing materials divided by the effective input power. As the number of mesh elements increases, the NPA value tends to stabilize, namely, after NPA value reaches stability, the simulation results are considered accurate, and increasing the number of elements has no essential effect on the simulation results, and here we use NPA in the study of mesh independence. Figure 4 shows the relationship among the number of elements and NPA. In this study, the mesh of 101,780 elements (including 89,345 domain elements, 11,453 boundary elements and 982 edge elements.) was determined to be used for simulation.

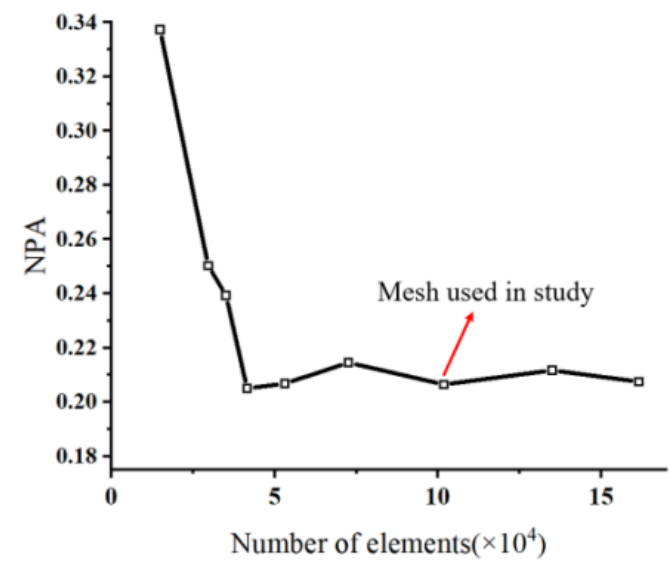

Figure 4. The relationship between the number of elements and NPA.

\subsection{Simulation Procedure}

After setting the implicit function and the parameters of the transformation optical layer, the distribution of the electric field can be computed. Since the modeling position of each physical region is always unchanged, after computing the electric field, it is essential to pull the dissipated power calculated by the electric field back to the modeling position (i.e., the initial position). It is worth noting that since the motion of the heated sample is translational motion, the coordinate transformation involved in the transformation of the dissipated power is only related to the $\mathrm{X}$ coordinate, which can be expressed as:

$$
\left\{\begin{array}{c}
x_{1}=x_{0}+v \cdot \Delta t \\
y_{1}=y_{0} \\
z_{1}=z_{0}
\end{array}\right.
$$

where $\left(x_{0}, y_{0}, z_{0}\right)$ are the initial coordinates of the sample and $\left(x_{1}, y_{1}, z_{1}\right)$ is the next time step position of the sample. After one time step $(\Delta t)$, the electric field distribution will be computed, and for calculating the temperature field, the dissipated power will be pulled back to the initial position. After that, the implicit function and parameters of the optical transformation layer are updated to move the sample to the next position for the computation of the next time step. The flowchart of the simulation is displayed in Figure 5. 


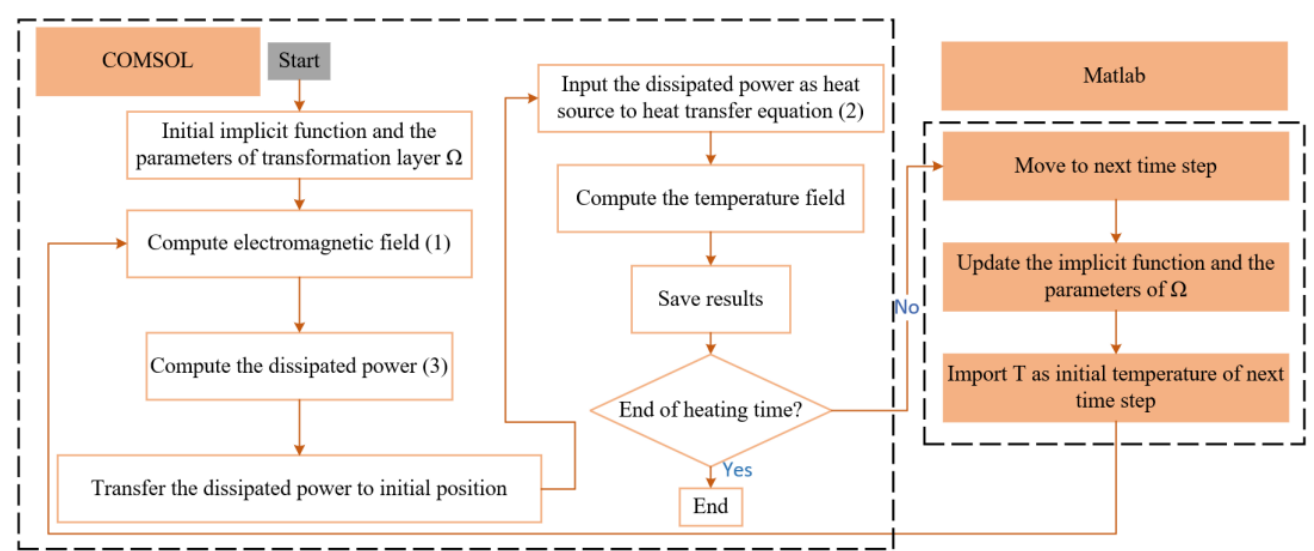

Figure 5. The flowchart of the simulation.

\section{Results and Discussion}

\subsection{Model Verification}

\subsubsection{Electromagnetic Field Verification}

In order to verify the correctness of the proposed method, the electromagnetic field distribution and port reflection coefficients calculated by the proposed method are compared with those calculated by the conventional method (i.e., simulated at discrete locations). It is assumed that the initial position of the heated sample is located at the leftmost part of the conveyor belt and moves to the rightmost part at a velocity of $10 \mathrm{~mm} / \mathrm{s}$. The cavity has an initial height of $150 \mathrm{~mm}$ and is stretched upward at a rate of $5 \mathrm{~mm} / \mathrm{s}$. The input microwave power is set to $100 \mathrm{~W}$.

As shown in Figure 6, the electric field distribution of a discrete position and simulated by the proposed method at $t=10 \mathrm{~s}$ are compared. It is observed that the electric field in the optical transformation layer is compressed to a certain extent, which is caused by the medium in the form of a tensor inside the optical transformation layer. In the cavity below the transformation layer, the electric field distribution simulated by the proposed method is consistent with the discrete position. It is worth mentioning that the model is built only once using the proposed method.

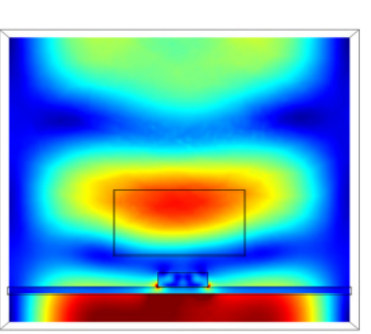

(a)

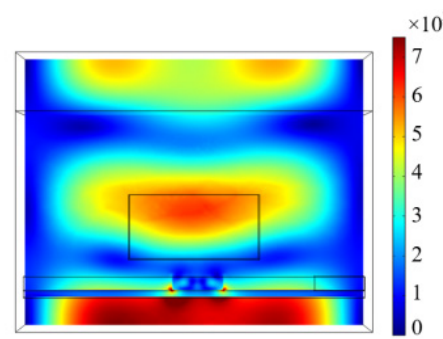

(b)

Figure 6. Electric field distribution at discrete height and transform height $(t=10 \mathrm{~s})$ : (a) discrete height; (b) proposed method.

Figure 7 shows the comparison diagram of the electric field distribution of the potato's center cross section simulated by the proposed method and at the discrete location simulated by the conventional method at $t=10 \mathrm{~s}$; the two methods coincide well.

Moreover, we also calculated the port reflection coefficients $S_{11}$ at different times according to the assumed translation velocity and stretching rate, as shown in Figure 8. The results of the two calculations agree very well, which verify the correctness of the proposed method. 


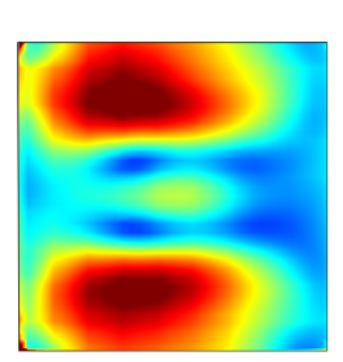

(a)

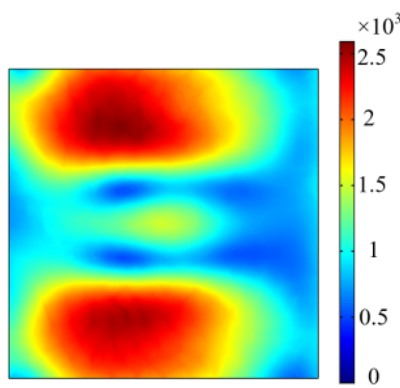

(b)

Figure 7. The comparison of electric field distribution at the center section of the potato $(t=10 \mathrm{~s})$ : (a) proposed method; (b) conventional method.

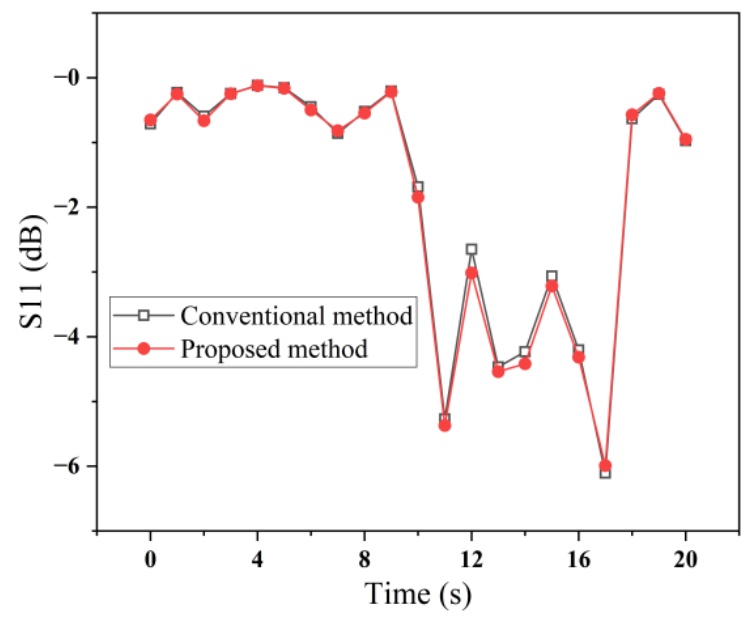

Figure 8. $\mathrm{S}_{11}$ computed by the proposed method and the conventional method.

By comparing the electric field and the reflection coefficient $S_{11}$, the correctness of the proposed method is verified.

\subsubsection{Experimental Verification}

To validate the proposed method, we build an experimental system, as shown in Figure 9. Figure 9a,b shows the sketch of the experimental system and actual experimental system, respectively. The microwave is fed by a microwave solid-state source generator at a frequency of $2.45 \mathrm{GHz}$ and a power of $150 \mathrm{~W}$ through a coaxial line fed into the waveguide connector. A circulator is used to ensure that the reflected wave is absorbed by the load to protect the microwave source. In order to measure the effective input power and reflection power, a microwave power meter is connected with a directional coupler through the power probes. Inside the microwave cavity, a block of potato is placed on the conveyor belt. The surface temperature image of a potato slice is recorded by a thermal imaging camera with an accuracy of $0.03 \mathrm{~K}$. During the microwave heating process, the fiber optic thermometer records the temperature of the fixed point of the potato. After heating, the microwave source is turned off and the potato is immediately removed from the microwave cavity and placed on the workbench. The thermal imaging camera then immediately photographs the temperature distribution on the upper surface of the potato. The total heating time is $20 \mathrm{~s}$.

Figure 10 shows the comparison of temperature distribution on the upper surface of potatoes between simulations and experiments, in which Figure 10a,b show the temperature distribution of potatoes heated for $20 \mathrm{~s}$ with the cavity height of $200 \mathrm{~mm}$ and $230 \mathrm{~mm}$, respectively, and the translation speed is $10 \mathrm{~mm} / \mathrm{s}$. Sample 1 and sample 2 correspond to one experiment, respectively. It can be seen that the temperature distribution of simulations and experiments are in good agreement. The temperature distribution in the experiments diverges slightly from the simulation results, and, as can be seen from the color legend 
(simulation: $20-70{ }^{\circ} \mathrm{C}$, experiment: $20-68{ }^{\circ} \mathrm{C}$ ), the experimental temperature is slightly lower than the simulation because there will be a 2 to $3 \mathrm{~s}$ delay in the process from heating to camera temperature measurement, which will cause a temperature divergence and temperature reduction. In general, the correctness of the simulation method can be verified by comparing the experimental results with the simulation results.

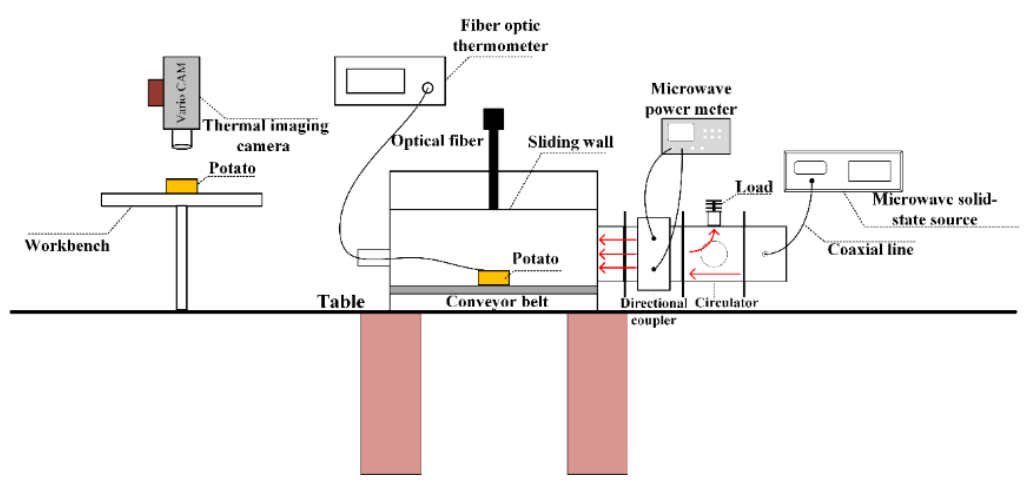

(a)

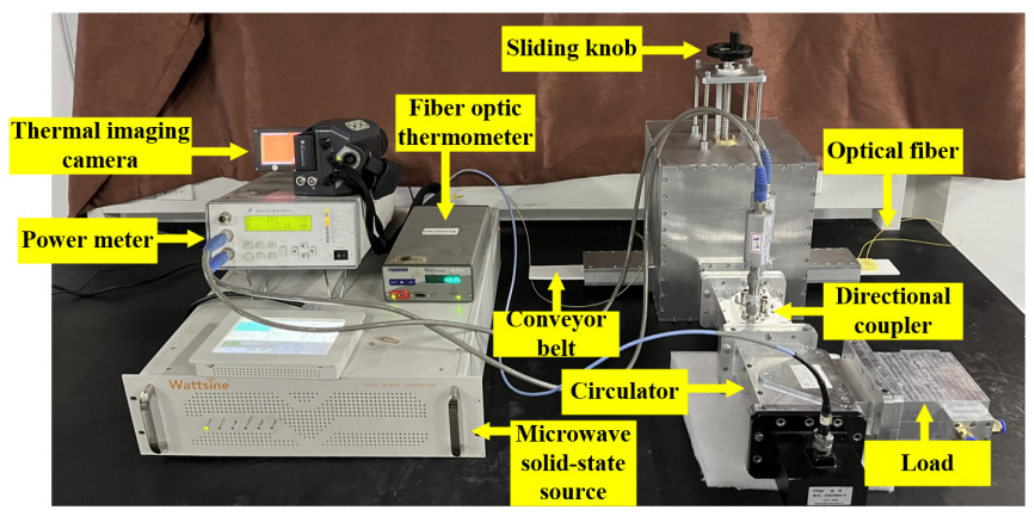

(b)

Figure 9. (a) Sketch of the experimental system; (b) actual experimental system.

(a)

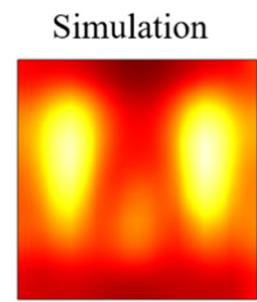

Simulation

(b)

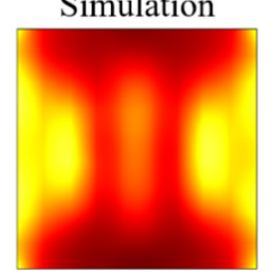

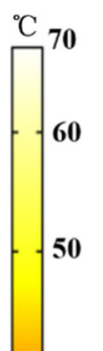

40

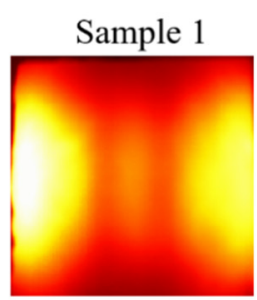

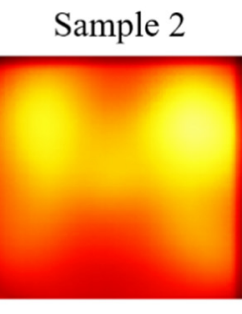

Sample 2

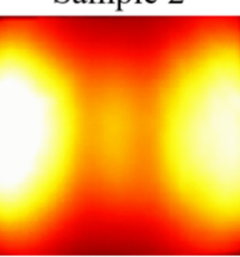

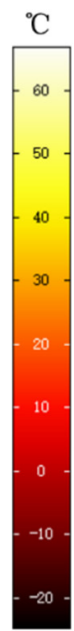

Figure 10. Temperature distribution of simulations and experiments on the upper surface of potatoes: (a) cavity height: $200 \mathrm{~mm}$; (b) cavity height: $230 \mathrm{~mm}$.

Figure 11 shows the point temperature variation curve of the central intercept point on the upper surface of the potatoes with time, and P1 represents the position of the tempera- 
ture measurement point. It is found that under the same conditions when potatoes move at $10 \mathrm{~mm} / \mathrm{s}$, a higher point temperature is obtained when the cavity height is $230 \mathrm{~mm}$ than when the cavity height is $200 \mathrm{~mm}$, which is consistent with the temperature distribution images on the upper surface of the potatoes. Most importantly, the experimental results agree well with the simulation results.

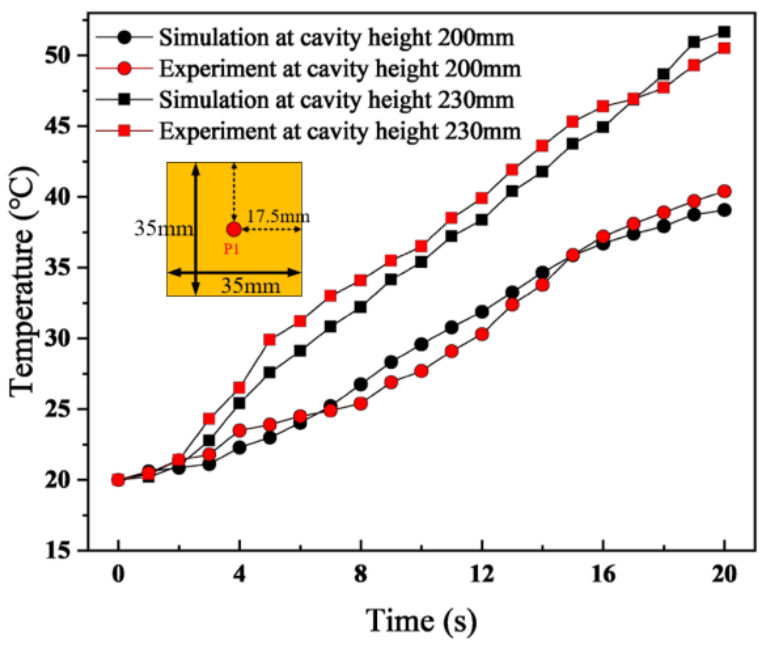

Figure 11. Comparison of point temperature obtained by simulations and experiments.

In general, the accuracy of the simulation method was verified by comparing the temperature distribution on the upper surface of potatoes and the point temperature at the center of the potato surface.

\subsection{Model Analysis}

\subsubsection{Uniformity and Efficiency Analysis}

Microwave heating is always accompanied by two important topics: heating efficiency and uniformity, they will be discussed below. Uniformity of microwave heating is defined by the coefficient of variation $(\mathrm{COV})$ of the heated sample:

$$
\operatorname{COV}=\frac{\sqrt{\frac{1}{N} \sum_{j=1}^{N}\left(T_{j}-\bar{T}\right)^{2}}}{\bar{T}-T_{0}}
$$

where $T_{j}$ denotes every mesh point temperature, $T_{0}$ denotes the initial body temperature of the sample, $\bar{T}$ and $N$ represent the average body temperature of the sample and the number of the mesh points of the sample domain, respectively. The smaller the coefficient of variation, the better the heating uniformity obtained. The heating efficiency can be measured by the port reflection coefficient $S_{11}$ or the average body temperature of the heated sample.

First, the heating efficiency is analyzed. Table 2 shows the average body temperature $\left(T_{a}\right)$ and coefficient of variation $(C O V)$ of the heated sample after $20 \mathrm{~s}$ of microwave heating under various conditions. The microwave input power is $200 \mathrm{~W}$. The $x$ is the coordinate of the center point of the potato block, and the potato is parallel to the conveyor belt. The $v$ is the speed at which the potato moves from the far left to the right on the belt. $T_{a}$ denotes the average body temperature of potatoes heated for $20 \mathrm{~s}$. 
Table 2. $T_{a}$ and COV of the heated sample for $20 \mathrm{~s}$ under different conditions.

\begin{tabular}{cccc}
\hline Cavity Height & Sample Location & $\boldsymbol{T}_{\boldsymbol{a}}$ & $\boldsymbol{C O V}$ \\
\hline $160 \mathrm{~mm}$ & $x=120 \mathrm{~mm}$ & $40.76^{\circ} \mathrm{C}$ & 0.539 \\
$160 \mathrm{~mm}$ & $v=10 \mathrm{~mm} / \mathrm{s}$ & $50.61{ }^{\circ} \mathrm{C}$ & 0.415 \\
$180 \mathrm{~mm}$ & $x=120 \mathrm{~mm}$ & $31.31^{\circ} \mathrm{C}$ & 0.688 \\
$180 \mathrm{~mm}$ & $v=10 \mathrm{~mm} / \mathrm{s}$ & $30.13^{\circ} \mathrm{C}$ & 0.409 \\
$200 \mathrm{~mm}$ & $x=120 \mathrm{~mm}$ & $47.26^{\circ} \mathrm{C}$ & 0.682 \\
$200 \mathrm{~mm}$ & $v=10 \mathrm{~mm} / \mathrm{s}$ & $64.67^{\circ} \mathrm{C}$ & 0.430 \\
$\Delta h=150-250 \mathrm{~mm}(5 \mathrm{~mm} / \mathrm{s})$ & $x=120 \mathrm{~mm}$ & $51.41{ }^{\circ} \mathrm{C}$ & 0.474 \\
$\Delta h=150-250 \mathrm{~mm}(5 \mathrm{~mm} / \mathrm{s})$ & $v=10 \mathrm{~mm} / \mathrm{s}$ & $47.16^{\circ} \mathrm{C}$ & 0.530 \\
\hline
\end{tabular}

As can be seen from Table 2, when the potato is fixed at the position $x=120 \mathrm{~mm}$ and the cavity height is different, the heating efficiency is different. When the cavity height is $160 \mathrm{~mm}, 180 \mathrm{~mm}$ and $200 \mathrm{~mm}$, the average body temperature of the potato is $40.76{ }^{\circ} \mathrm{C}, 31.31{ }^{\circ} \mathrm{C}$ and $47.26{ }^{\circ} \mathrm{C}$, respectively. When the potato translates at $10 \mathrm{~mm} / \mathrm{s}$, the average body temperatures of the potato heated for $20 \mathrm{~s}$ are $50.61{ }^{\circ} \mathrm{C}, 30.13{ }^{\circ} \mathrm{C}$ and $64.67^{\circ} \mathrm{C}$ respectively, indicating that translational motion can improve the heating efficiency in most cases, and the improvement range is $-3.8 \%$ to $36.8 \%$. Notice that when the height of the cavity is $180 \mathrm{~mm}$, the translational motion of potato does not improve the heating efficiency, time is used as a variable to carry out parametric scanning of $S_{11}$, and the results are shown in Figure 12. It is found that when the height of the cavity is $180 \mathrm{~mm}$, the $\mathrm{S}_{11}$ at each time is relatively high and the energy utilization rate is low which also explains why translational motion does not improve heating efficiency at $180 \mathrm{~mm}$ cavity height. In fact, due to translational motion enabling the potato to traverse more positions, the effects of microwave heating on those positions with a high port reflection coefficient is reduced, thus improving the heating efficiency to a certain extent.

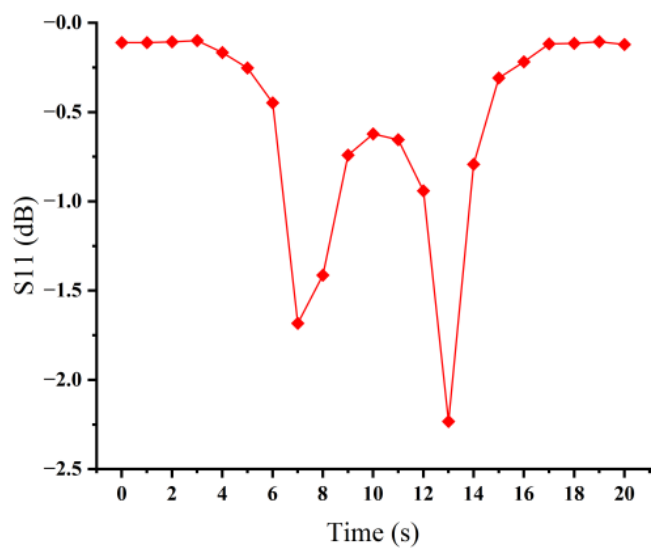

Figure 12. Port reflection coefficient at different times. (Cavity height: $180 \mathrm{~mm}$ ).

When the upper wall of the cavity is stretching at $5 \mathrm{~mm} / \mathrm{s}$ within the range of $150 \mathrm{~mm}$ to $250 \mathrm{~mm}$, the average body temperature of the potato fixed at $x=120 \mathrm{~mm}$ is $51.41{ }^{\circ} \mathrm{C}$ after heating for $20 \mathrm{~s}$. Compared with the fixed position of the potato and the fixed height of the cavity, the heating efficiency is improved, indicating that stretching motion can also improve the heating efficiency.

The uniformity of heating is then analyzed. As can be seen from Table 2, when the potato position is $x=120 \mathrm{~mm}$ and the cavity height is $160 \mathrm{~mm}, 180 \mathrm{~mm}$ and $200 \mathrm{~mm}$, the coefficient of variation is $0.539,0.688$ and 0.682 , respectively, which are relatively large values, indicating poor heating uniformity. When the potato translates at $10 \mathrm{~mm} / \mathrm{s}$, the $C O V$ at different cavity heights is $0.415,0.409$ and 0.430 , respectively, indicating that translational movement can improve heating uniformity; the improvement range is $23.0 \%$ to $40.6 \%$. Figure 13 shows the temperature distribution of the potato fixed at $x=120 \mathrm{~mm}$ or translated 
at $10 \mathrm{~mm} / \mathrm{s}$ for $20 \mathrm{~s}$ with different cavity heights. As the distribution of the electric field inside the potato changes at any time due to the translational motion, it is no longer a single electric field, so the improvement of heating uniformity is obvious. Figure 14 shows the electric field distribution of the potato at different motion moments with the height of the cavity of $160 \mathrm{~mm}$.

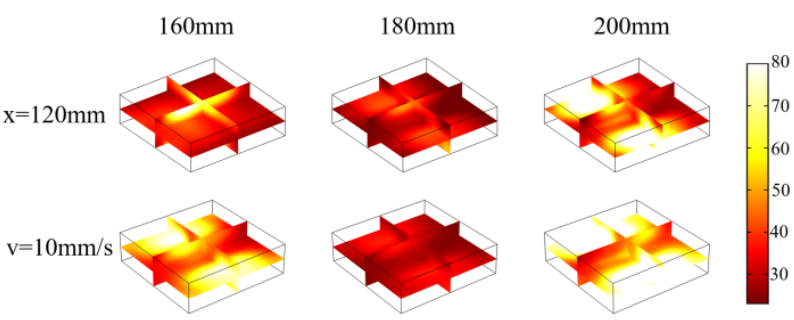

Figure 13. Temperature distribution comparison of potato fixed heating at $x=120 \mathrm{~mm}$ and translating at $10 \mathrm{~mm} / \mathrm{s}$ (unit: ${ }^{\circ} \mathrm{C}$ ).
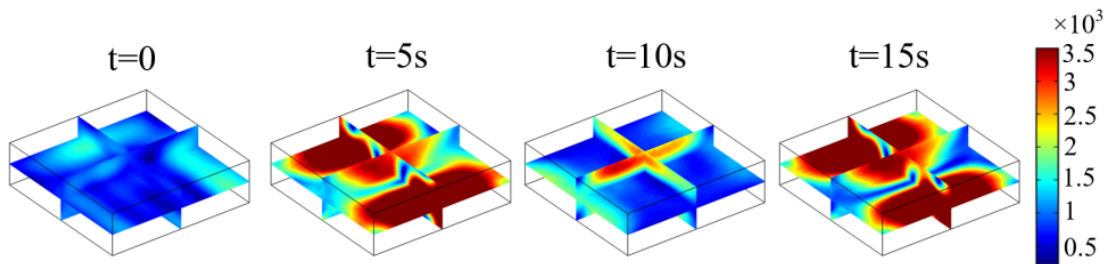

Figure 14. Electric field distribution of potato at different motion moments (Cavity height: $160 \mathrm{~mm}$, unit: $\mathrm{V} / \mathrm{m})$.

When the position of the potato is fixed at $x=120 \mathrm{~mm}$ and the upper wall of the microwave cavity is stretched, the COV of the potato obtained after simulation is 0.474 . Compared with the fixed potato position and the fixed cavity height, the uniformity of heating is improved by $12.1 \%$ to $31.1 \%$. Figure 15 shows the electric field distribution norm of the potato at different cavity heights. It can be seen that the electric field norm inside the potatoes is different at different cavity heights, which is also the reason why stretching improves heating uniformity.

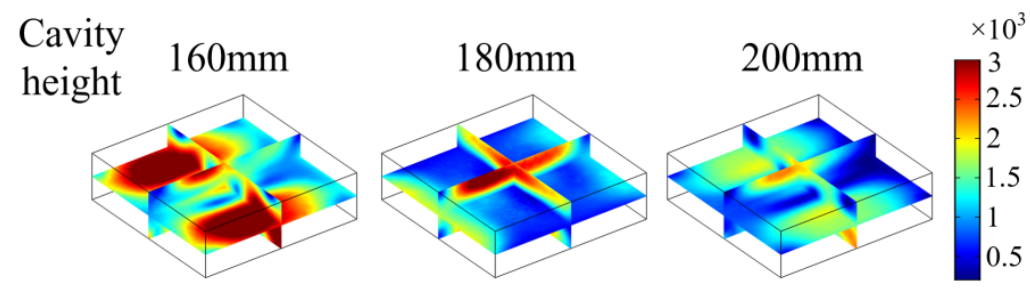

Figure 15. Electric field distribution of the potato at different cavity heights. (unit: $\mathrm{V} / \mathrm{m}$ ).

Moreover, we also simulate the microwave heating process of the stretching motion of the upper wall of the cavity and the translational motion of the potato at the same time. The stretching speed is $5 \mathrm{~mm} / \mathrm{s}$, the stretching range is $150 \mathrm{~mm}$ to $250 \mathrm{~mm}$, the translation speed is $10 \mathrm{~mm} / \mathrm{s}$, and the total heating time is $20 \mathrm{~s}$. The results show that the average body temperature of potato after heating is $47.16^{\circ} \mathrm{C}$, and the $\mathrm{COV}$ is 0.530 . Compared with fixed heating, the heating efficiency is improved from $0.2 \%$ to $50.6 \%$, and the heating uniformity is improved from $1.7 \%$ to $23.0 \%$. It can be seen that the joint action of stretching and translational motion improves the efficiency of microwave heating under certain conditions and the heating uniformity within a certain range. 


\subsubsection{Select Location Heating}

In general, microwave heating requires both high efficiency and high uniformity. The heating efficiency is high when the port reflection coefficient is small, but it is difficult to get good heating uniformity when heating in one fixed position. Therefore, by adjusting the height of the microwave cavity and the position of the potato on the conveyor belt, 8 positions with low reflection coefficients of port are selected for select location heating by taking advantage of the characteristics of the proposed microwave cavity, as shown in Table 3. Heating is from top to bottom according to the position and time in Table 3. The microwave input power is $200 \mathrm{~W}$ and the total heating time is $20 \mathrm{~s}$. The heating time for each position is also shown in Table 3 and the results after heating at select locations are shown in Table 4 . The results show that the heating efficiency is greatly improved by selecting location heating, and the range of improvement is $96.7 \%$ to $196.8 \%$ compared with fixed heating, $43.7 \%$ to $208.5 \%$ compared with only translational motion, $80.8 \%$ compared with only stretching motion and $97.1 \%$ compared with joint translational motion and stretching motion. Moreover, the uniformity of heating is also improved. Compared with fixed heating, the range of improvement is $33.2 \%$ to $47.7 \%$; compared with only translation, the range of improvement is $12.0 \%$ to $16.3 \%$; compared with only stretching motion, the range of improvement is $24.1 \%$; compared with the joint translation and stretching motion, the improvement is $32.1 \%$.

Table 3. The $\mathrm{S}_{11}$ and heating time at 8 select locations (material: potato).

\begin{tabular}{cccc}
\hline Cavity Height & Sample Location & $\mathbf{S}_{\mathbf{1 1}}$ & Heating Time \\
\hline $155 \mathrm{~mm}$ & $x=118 \mathrm{~mm}$ & $-5.60 \mathrm{~dB}$ & $2 \mathrm{~s}$ \\
$205 \mathrm{~mm}$ & $x=96 \mathrm{~mm}$ & $-8.92 \mathrm{~dB}$ & $2 \mathrm{~s}$ \\
$210 \mathrm{~mm}$ & $x=110 \mathrm{~mm}$ & $-10.87 \mathrm{~dB}$ & $3 \mathrm{~s}$ \\
$210 \mathrm{~mm}$ & $x=105 \mathrm{~mm}$ & $-4.65 \mathrm{~dB}$ & $2 \mathrm{~s}$ \\
$220 \mathrm{~mm}$ & $x=147 \mathrm{~mm}$ & $-9.45 \mathrm{~dB}$ & $2 \mathrm{~s}$ \\
$220 \mathrm{~mm}$ & $x=37 \mathrm{~mm}$ & $-14.19 \mathrm{~dB}$ & $3 \mathrm{~s}$ \\
$230 \mathrm{~mm}$ & $x=48 \mathrm{~mm}$ & $-13.35 \mathrm{~dB}$ & $3 \mathrm{~s}$ \\
$230 \mathrm{~mm}$ & $x=196 \mathrm{~mm}$ & $-11.51 \mathrm{~dB}$ & $3 \mathrm{~s}$ \\
\hline
\end{tabular}

Table 4. $T_{a}$ and $C O V$ after heating at select locations (material: potato).

\begin{tabular}{ccc}
\hline Heating Method & $T_{a}$ & COV \\
\hline Select locations heating & $92.95^{\circ} \mathrm{C}$ & 0.36 \\
\hline
\end{tabular}

The results show that select location heating can enhance the heating efficiency and uniformity at the same time, and the improvement of heating efficiency is very significant.

\subsubsection{Other Materials}

In order to verify whether the proposed microwave cavity structure is suitable for other materials, we also simulate the microwave heating process of beef and distilled water as the load. As shown in Table 5, beef is used as the heating material in different locations for select location heating and fixed heating, and the input power is $200 \mathrm{~W}$. It can be seen from Table 6 that the average body temperature of beef increased from $50.13^{\circ} \mathrm{C}$ to $83.72{ }^{\circ} \mathrm{C}$ with an improvement rate of $40.1 \%$, the COV decreased from 0.695 to 0.561 , and the heating uniformity improved by $19.3 \%$. 
Table 5. The select heating locations and heating time of beef.

\begin{tabular}{cccc}
\hline Cavity Height & Sample Location & $\mathbf{S}_{\mathbf{1 1}}$ & Heating Time \\
\hline $150 \mathrm{~mm}$ & $x=78 \mathrm{~mm}$ & $-5.21 \mathrm{~dB}$ & $3 \mathrm{~s}$ \\
$210 \mathrm{~mm}$ & $x=106 \mathrm{~mm}$ & $-7.29 \mathrm{~dB}$ & $3 \mathrm{~s}$ \\
$220 \mathrm{~mm}$ & $x=25 \mathrm{~mm}$ & $-8.80 \mathrm{~dB}$ & $4 \mathrm{~s}$ \\
$220 \mathrm{~mm}$ & $x=37 \mathrm{~mm}$ & $-22.95 \mathrm{~dB}$ & $4 \mathrm{~s}$ \\
$230 \mathrm{~mm}$ & $x=35 \mathrm{~mm}$ & $-9.49 \mathrm{~dB}$ & $3 \mathrm{~s}$ \\
$240 \mathrm{~mm}$ & $x=79 \mathrm{~mm}$ & $-5.54 \mathrm{~dB}$ & $3 \mathrm{~s}$ \\
\hline
\end{tabular}

Table 6. $T_{a}$ and COV after heating at select locations (material: beef).

\begin{tabular}{ccc}
\hline Heating Method & $T_{a}$ & COV \\
\hline Select locations heating & $83.72{ }^{\circ} \mathrm{C}$ & 0.561 \\
Fixed heating & $50.13^{\circ} \mathrm{C}$ & 0.695 \\
\hline
\end{tabular}

Table 7 shows the select locations of distilled water as the heated material, and as shown in Table 8, the average body temperature of distilled water increased from $41.59{ }^{\circ} \mathrm{C}$ to $90.79{ }^{\circ} \mathrm{C}$ after heating at select locations, reaching an improvement rate of $118.3 \%$, and the heating uniformity also improves by 45.9\%. Moreover, Figures 16 and 17 show the comparison of $\mathrm{COV}$ and average body temperature of potato, beef and distilled water under select location heating and fixed heating, respectively.

Table 7. The select heating locations and heating time of distilled water.

\begin{tabular}{cccc}
\hline Cavity Height & Sample Location & $\mathbf{S}_{\mathbf{1 1}}$ & Heating Time \\
\hline $150 \mathrm{~mm}$ & $x=87 \mathrm{~mm}$ & $-6.58 \mathrm{~dB}$ & $3 \mathrm{~s}$ \\
$200 \mathrm{~mm}$ & $x=87 \mathrm{~mm}$ & $-5.15 \mathrm{~dB}$ & $3 \mathrm{~s}$ \\
$210 \mathrm{~mm}$ & $x=127 \mathrm{~mm}$ & $-24.92 \mathrm{~dB}$ & $3 \mathrm{~s}$ \\
$220 \mathrm{~mm}$ & $x=37 \mathrm{~mm}$ & $-22.03 \mathrm{~dB}$ & $2 \mathrm{~s}$ \\
$220 \mathrm{~mm}$ & $x=47 \mathrm{~mm}$ & $-7.49 \mathrm{~dB}$ & $3 \mathrm{~s}$ \\
$230 \mathrm{~mm}$ & $x=37 \mathrm{~mm}$ & $-9.26 \mathrm{~dB}$ & $3 \mathrm{~s}$ \\
$230 \mathrm{~mm}$ & $x=47 \mathrm{~mm}$ & $-8.15 \mathrm{~dB}$ & $3 \mathrm{~s}$ \\
\hline
\end{tabular}

Table 8. Ta and COV after heating at select locations (material: distilled water).

\begin{tabular}{ccc}
\hline Heating Method & $T_{a}$ & $C O V$ \\
\hline Select locations heating & $90.79{ }^{\circ} \mathrm{C}$ & 0.482 \\
Fixed heating & $41.59{ }^{\circ} \mathrm{C}$ & 0.703 \\
\hline
\end{tabular}

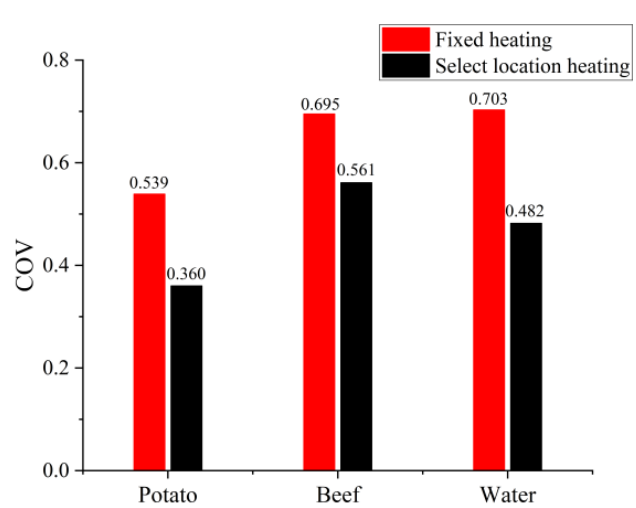

Figure 16. Comparison of $\mathrm{COV}$ of different materials in select locations heating and fixed heating. 


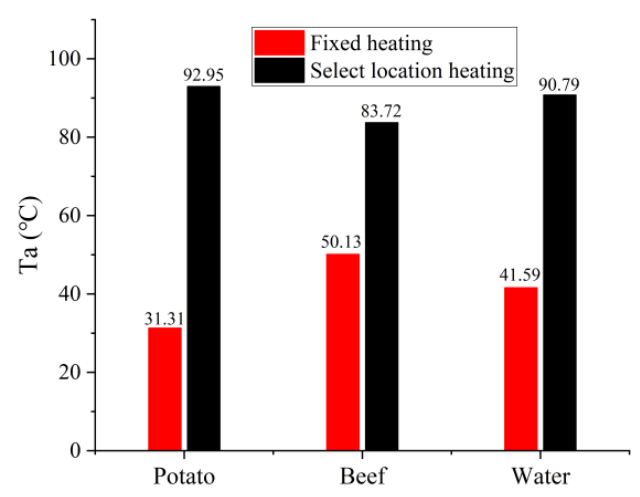

Figure 17. Comparison of body average temperature of different materials in select location heating and fixed heating.

The results show that the proposed microwave structure can enhance the heating efficiency and heating uniformity for different materials, which verifies the universal applicability of the proposed model.

\section{Conclusions}

This paper presents a microwave oven with an upper wall stretching structure and conveyor belt to improve the uniformity and efficiency of microwave heating. The method of transformation optics is used to calculate the stretching motion of the upper wall of the microwave oven, and the implicit function method is used to compute the translational motion of the conveyor belt. Experiments are carried out to verify the correctness, and the two methods can be coupled well. By the use of the proposed microwave oven model, heating efficiency and heating uniformity under different conditions were studied. The results show that both motion modes can enhance heating efficiency and heating uniformity. In addition, select location heating is studied. By selecting the positions with small reflection coefficients for multi-point heating, the heating efficiency is greatly improved and the heating uniformity is improved. In order to verify the universal applicability of the proposed microwave oven structure, the performance of other materials is studied, and the proposed cavity can be applied to other materials. In addition, this paper provides a feasible demonstration for the combination of the transformation optics method and the implicit function method and provides a promising solution tool for microwave heating models with other complex motion forms.

Author Contributions: H.G. designed the simulation model, carried out the simulation of the proposed model, carried out the experiments, analyzed the data, and wrote the initial draft of the manuscript; G.W., J.Y. and X.Y. conceived and designed the experiments; J.Y., H.Z. and Y.Y. reviewed and contributed to the final manuscript. All authors have read and agreed to the published version of the manuscript.

Funding: This work was supported by Sichuan Science and Technology Program under Grant 2021YFG0265, the Sichuan University and Panzhihua City project (Grant No. 2019CDPZH-20) and the Sichuan University and Suining City project (Grant No. 2020CDSN-01).

Institutional Review Board Statement: Not applicable.

Informed Consent Statement: Not applicable.

Data Availability Statement: All data and models generated or used during the study appear in the submitted article.

Conflicts of Interest: The authors declare no conflict of interest.

\section{References}

1. Adam, D. Microwave chemistry: Out of the kitchen. Nature 2003, 421, 571-572. [CrossRef] [PubMed]

2. Datta, A.K. Handbook of Microwave Technology for Food Application; CRC Press: Boca Raton, FL, USA, 2001 ; p. 6. 
3. Mingos, D.M.P. The applications of microwaves in chemical syntheses. Res. Chem. Intermed. 1994, 20, 85-91. [CrossRef]

4. Vadivambal, R.; Jayas, D.S. Non-uniform temperature distribution during microwave heating of food materials-A review. Food Bioprocess Technol. 2010, 3, 161-171. [CrossRef]

5. Yi, Q.; Lan, J.; Ye, J.; Zhu, H.; Yang, Y.; Wu, Y.; Huang, K. A simulation method of coupled model for a microwave heating process with multiple moving elements. Chem. Eng. Sci. 2021, 231, 116339. [CrossRef]

6. Ye, J.; Xia, Y.; Yi, Q.; Zhu, H.; Yang, Y.; Huang, K.; Shi, K. Multiphysics modeling of microwave heating of solid samples in rotary lifting motion in a rectangular multi-mode cavity. Innov. Food Sci. Emerg. Technol. 2021, 73, 102767. [CrossRef]

7. Luan, D.; Tang, J.; Pedrow, P.D.; Liu, F.; Tang, Z. Analysis of electric field distribution within a microwave assisted thermal sterilization (MATS) system by computer simulation. J. Food Eng. 2016, 188, 87-97. [CrossRef]

8. Zhu, H.; He, J.; Hong, T.; Yang, Q.; Wu, Y.; Yang, Y.; Huang, K. A rotary radiation structure for microwave heating uniformity improvement. Appl. Therm. Eng. 2018, 141, 648-658. [CrossRef]

9. Li, Z.Y.; Wang, R.F.; Kudra, T. Uniformity issue in microwave drying. Dry. Technol. 2011, 29, 652-660. [CrossRef]

10. Xiong, G.; Zhu, H.; Huang, K.; Yang, Y.; Fan, Z.; Ye, J. The impact of pins on dual-port microwave heating uniformity and efficiency with dual frequency. J. Microw. Power Electromagn. Energy 2020, 54, 83-98. [CrossRef]

11. Ye, J.; Zhang, C.; Zhu, H. A Temperature-Control System for Continuous-Flow Microwave Heating Using a Magnetron as Microwave Source. IEEE Access 2020, 8, 44391-44399. [CrossRef]

12. Hong, Y.D.; Lin, B.Q.; Li, H.; Dai, H.M.; Zhu, C.J.; Yao, H. Three-dimensional simulation of microwave heating coal sample with varying parameters. Appl. Therm. Eng. 2016, 93, 1145-1154. [CrossRef]

13. Bae, S.H.; Jeong, M.G.; Kim, J.H.; Lee, W.S. A continuous power-controlled microwave belt drier improving heating uniformity. IEEE Microw. Wirel. Compon. Lett. 2017, 27, 527-529. [CrossRef]

14. Itaya, Y.; Uchiyama, S.; Hatano, S.; Mori, S. Effect of scattering by fluidization of electrically conductive beads on electrical field intensity profile in microwave dryers. Dry. Technol. 2005, 23, 273-287. [CrossRef]

15. Wang, R.; Huo, H.; Dou, R.; Li, Z.; Mujumdar, A.S. Effect of the Inside Placement of Electrically Conductive Beads on Electric Field Uniformity in a Microwave Applicator. Dry. Technol. 2014, 32, 1997-2004. [CrossRef]

16. Plaza-gonzález, P.; Monzó-cabrera, J.; Catalá-civera, J.M.; Sánchez-hernández, D. New Approach for the Prediction of the Electric Field Distribution in Multimode Micwave-Heating Applicators with Mode Stirrers. IEEE Trans. Magn. 2004, 40, 1672-1678. [CrossRef]

17. Ryynänen, S.; Ohlsson, T. Microwave heating uniformity of ready meals as affected by placement, composition, and geometry. J. Food Sci. 1996, 61, 620-624. [CrossRef]

18. Geedipalli, S.S.R.; Rakesh, V.; Datta, A.K. Modeling the heating uniformity contributed by a rotating turntable in microwave ovens. J. Food Eng. 2007, 82, 359-368. [CrossRef]

19. Zhou, R.; Yang, X.; Sun, D.; Jia, G. Multiple tube structure for heating uniformity and efficiency optimization of microwave ovens. EPJ Appl. Phys. 2015, 69, 20201. [CrossRef]

20. Raaholt, B.W.; Isaksson, S.; Hamberg, L.; Fhager, A.; Hamnerius, Y. Continuous tubular microwave heating of homogeneous foods: Evaluation of heating uniformity. J. Microw. Power Electromagn. Energy 2016, 50, 43-65. [CrossRef]

21. Salema, A.A.; Afzal, M.T. Numerical simulation of heating behaviour in biomass bed and pellets under multimode microwave system. Int. J. Therm. Sci. 2015, 91, 12-24. [CrossRef]

22. Soto-Reyes, N.; Temis-Pérez, A.L.; López-Malo, A.; Rojas-Laguna, R.; Sosa-Morales, M.E. Effects of Shape and Size of Agar Gels on Heating Uniformity During Pulsed Microwave Treatment. J. Food Sci. 2015, 80, 1021-1025. [CrossRef] [PubMed]

23. Plaza-González, P.; Monzó-Cabrera, J.; Catalá-Civera, J.M.; Sánchez-Hernández, D. Effect of mode-stirrer configurations on dielectric heating performance in multimode microwave applicators. IEEE Trans. Microw. Theory Tech. 2005, 53, 1699-1705. [CrossRef]

24. Ma, W.; Hong, T.; Xie, T.; Wang, F.; Luo, B.; Zhou, J.; Yang, Y.; Zhu, H.; Huang, K. Simulation and analysis of oleic acid pretreatment for microwave-assisted biodiesel production. Processes 2018, 6, 142. [CrossRef]

25. He, J.; Yang, Y.; Zhu, H.; Li, K.; Yao, W.; Huang, K. Microwave heating based on two rotary waveguides to improve efficiency and uniformity by gradient descent method. Appl. Therm. Eng. 2020, 178, 115594. [CrossRef]

26. Liu, S.; Fukuoka, M.; Sakai, N. A finite element model for simulating temperature distributions in rotating food during microwave heating. J. Food Eng. 2013, 115, 49-62. [CrossRef]

27. Wu, Y.; Yan, B.; Yang, Y.; Zhu, H.; Huang, K. Accordion microwave oven for uniformity and efficiency heating. Int. J. RF Microw. Comput. Eng. 2020, 30, e22190. [CrossRef]

28. Zhou, J.; Yang, X.; Ye, J.H.; Zhu, H.; Yuan, J.; Li, X.; Huang, K. Arbitrary Lagrangian-Eulerian method for computation of rotating target during microwave heating. Int. J. Heat Mass Transf. 2019, 134, 271-285. [CrossRef]

29. Milton, G.W.; Briane, M.; Willis, J.R. On cloaking for elasticity and physical equations with a transformation invariant form. New J. Phys. 2006, 8, 248. [CrossRef]

30. Ward, A.J.; Pendry, J.B. Refraction and geometry in Maxwell's equations. J. Mod. Opt. 2009, 43, 37-41. [CrossRef]

31. Ye, J.H.; Zhu, H.C.; Liao, Y.H.; Zhou, Y.P.; Huang, K.M. Implicit function and level set methods for computation of moving elements during microwave heating. IEEE Trans. Microw. Theory Tech. 2017, 65, 4773-4784. [CrossRef] 